\title{
Prevalência da pancreatite crônica em pacientes portadores de cirrose hepática alcoólica: estudo histopatológico
}

\section{Prevalence of chronic pancreatitis in patients with alcoholic liver cirrhosis: histopathological study}

Júlia de Fátima Gonçalves Santos', Ademir Rocha², Luiz Carlos Marques de Oliveira²

\section{unitermos \\ Cirrose hepática alcoólica}

Pancreatite crônica

Álcool

Histopatologia

\section{resumo}

Introdução: O abuso crônico do álcool é importante causa de cirrose hepática e de pancreatite crônica. Objetivo: Avaliar a freqüência de pancreatite crônica (PC) em pacientes portadores de cirrose hepática alcoólica (CHA) por exame histopatológico. Casuística: Analisaram-se necropsias de 18 homens e sete mulheres portadores de CHA, com idade média e desvio padrão de 47,2 $\pm 13,8$ anos (24 a 83 anos), que consumiam em média $239,6 \pm 155,3 \mathrm{~g}$ de etanol/dia por um período médio de $16,7 \pm 7,2$ anos. Métodos: $\mathrm{O}$ diagnóstico histopatológico de cirrose hepática baseou-se na fibrose sistematizada com formação de septos delimitando nódulos, associada à inflamação crônica inespecífica. O diagnóstico histológico de PC baseou-se na identificação de exsudato mononuclear (EM) em torno dos ductos e no tecido conjuntivo inter e intralobular, acompanhado de traves de fibrose. A PC foi classificada como leve quando havia discreto EM em região periductal, interlobular ou intralobular acompanhado por finas traves de fibrose; moderada, pelo aumento da intensidade do exsudato e da fibrose, ocasionalmente associado com pequena dilatação dos ductos; a forma grave caracterizou-se pelo EM, densa fibrose, presença de rolhas, dilatação dos ductos, atrofia glandular e, às vezes, calcificação. Resultados: Observou-se PC em 20 pacientes portadores de CHA (80\%), que foi leve em 13 (52\%), moderada em dois (8\%) e grave em cinco (20\%). Conclusão: A pancreatite crônica está freqüentemente associada à CHA, sendo histologicamente grave em aproximadamente $1 / 4$ dos casos.
Introduction: The chronic alcohol abuse is an important cause of liver cirrhosis and chronic pancreatitis. Objective: To evaluate the prevalence of chronic pancreatitis (CP) in patients with alcoholic liver cirrhosis (ALC) based on histopathological assays. Subjects: Autopsies of 25 patients with ALC, 18 men and seven women, age ranging from 24 to 83 years (mean \pm standard deviation: $47.2 \pm 13.8$ ) were retrospectively analyzed. They ingested on average $239.6 \pm 155.3 \mathrm{~g}$ ethanol/day for a mean period of $16.7 \pm 7.2$ years. Methods: Liver cirrhosis histopathological diagnosis was based on the findings of systemized fibrosis with formation of septum-delimited nodules and nonspecific chronic inflammation. CP histopathological diagnosis was based on identification of mononuclear cell infiltration (MCI) around the ducts and in the inter-and intra-lobular connective tissue and presence of strands of fibrosis. CP was classified as mild (discrete $\mathrm{MCl}$ in the periductal, inter- or intra-lobular areas with thin strands of fibrosis), moderate (increased exudates and fibrosis, occasionally associated with small dilatation of ducts), and severe (evident $\mathrm{MCl}$, dense fibrosis, presence of plugs in the ducts, irregular ductal dilatation, glandular atrophy and, sometimes, calcification). Results: $C P$ was observed in 20 patients (80\%) with $A L C$, and was considered mild in 13 (52\%), moderate in two (8\%) and severe in five (20\%) patients. Conclusion: $C P$ is often associated with the $A L C$, and it can be histologically severe in approximately $1 / 4$ of the cases. key words

Alcoholic liver cirrhosis

Chronic pancreatitis

Alcohol

Histopathology

\footnotetext{
1. Médica do Serviço de Gastroenterologia do Hospital de Clínicas da Universidade Federal de Uberlândia (HC/UFU).

2. Professores do Departamento de Clínica Médica da Faculdade de Medicina da UFU.

Trabalho realizado no Serviço de Anatomia Patológica do HC/UFU.

Apoio financeiro: Coordenação de Aperfeiçoamento de Pessoal de Nível Superior (CAPES) e Fundação de Assistência, Ensino e Pesquisa de Uberlândia (FAEPU).

Este trabalho faz parte da dissertação de mestrado intitulada "Prevalência da pancreatite crônica em pacientes portadores de cirrose hepática alcoólica: estudo histopatológico", realizada no curso de mestrado em Ciências da Saúde da Faculdade de Medicina da UFU, defendida no dia 16 de setembro de 2005.
} 


\section{Introdução}

O álcool é fator etiológico comum tanto da cirrose hepática $(\mathrm{CH})^{(12,16)}$ quanto da pancreatite crônica (PC) ${ }^{(2,21)}$. O tempo médio de consumo alcoólico necessário para o desenvolvimento de PC tem sido descrito como menor do que para a $\mathrm{CH}^{(13,23,28)}$, todavia o tempo mínimo de alcoolismo para que cada uma delas ocorra é muito variável, sendo descrito de cinco $^{(31)}$ a $12^{(30)}$ anos para a PC e de oito ${ }^{(25,38)}$ a $20^{(17)}$ anos para a $\mathrm{CH}$. Em relação à quantidade de ingestão diária de etanol necessária para o desenvolvimento de ambas as doenças, também têm sido relatados valores variáveis, mas, de forma geral, se aceita que 60-80 g de etanol para homens e 20-40 $\mathrm{g}$ para mulheres são suficientes para o desenvolvimento da cirrose hepática alcoólica $(\mathrm{CHA})^{(16,37)}$, e 40-50 gramas para o de pancreatite crônica alcoólica (PCA) ${ }^{(19)}$.

Assim, como o tempo de ingestão etílica para o aparecimento de PC é geralmente menor do que aquele para a $\mathrm{CH}$, esperar-se-ia que a maioria dos pacientes portadores de CHA tivesse concomitantemente PCA, caso o tempo de alcoolismo fosse o único fator a ser levado em consideração. Entretanto, nem todos os indivíduos que abusam da ingestão alcoólica desenvolvem lesões importantes no fígado ou no pâncreas. A incidência de cirrose hepática entre os alcoolistas pesados é de cerca de $10 \%$ a $15 \%{ }^{(38)}$, e fatores adicionais para o surgimento da CHA incluiriam o sexo feminino(29,37), a concomitância de infecções por vírus hepatotrópicos ${ }^{(41)}$, fatores genéticos ${ }^{(14,33)} \mathrm{e}$, talvez, o aspecto nutricional ${ }^{(9)}$. No caso da PCA, que se desenvolveria em menos de $10 \%$ dos etilistas crônicos $^{(11)}$, outros fatores contribuintes seriam os genéticos ${ }^{(18,20)}$, os nutricionais ${ }^{(13,19)}$ e, talvez, o tabagismo ${ }^{(5)}$.

A freqüência de identificação da concomitância dessas duas entidades depende essencialmente do método utilizado para o diagnóstico da PC: variou de $7,7 \%^{(10)}$ a $63,6 \%^{(32)}$ quando se utilizou a colangiopancreatografia endoscópica retrógrada (CPER); de $17,3 \%{ }^{(40)}$ a $40 \%{ }^{(2)}$ usando-se testes funcionais com secretina-colecistocinina ( $\mathrm{S}-\mathrm{C})$, e de $8,9 \%{ }^{(22)}$ a $46 \%{ }^{(39)}$ quando se fez avaliação histológica pancreática.

$O$ presente estudo tem a finalidade de avaliar a freqüência da associação entre CHA e PCA em nosso meio, através do exame histopatológico do fígado e do pâncreas obtidos em necropsias de indivíduos portadores de cirrose hepática alcoólica.

\section{Casuística e métodos}

Foram revistas necropsias de 25 pacientes portadores de cirrose hepática alcoólica no Hospital de Clínicas da
Universidade Federal de Uberlândia (HC/UFU), realizadas no período de janeiro de 1990 a dezembro de 2003. Dezoito (72\%) eram homens e sete (28\%), mulheres, com idades entre 24 e 83 anos (média e desvio padrão de 47,2 \pm 13,8). A análise de seus prontuários médicos mostrou que nenhum paciente tinha diagnóstico de pancreatite, aguda ou crônica, e que a ingestão média diária de etanol foi $239,6 \pm$ $155,3 \mathrm{~g}$, por um período médio de $16,7 \pm 7,2$ anos. Para o cálculo da ingestão de etanol puro foi considerado que, para a cachaça, uma dose de $50 \mathrm{ml}$, uma garrafa de 660 $\mathrm{ml}$ e um litro da bebida, correspondem a $17 \mathrm{~g}, 220 \mathrm{~g}$ e $340 \mathrm{~g}$ de etanol, respectivamente. Para outros destilados (conhaque), considerou-se a dose de $50 \mathrm{ml}$ equivalente a $16 \mathrm{~g}$ de etanol puro; em relação à cerveja, cada garrafa de $660 \mathrm{ml}$ contém $25 \mathrm{~g}$ de etanol(27).

Os fragmentos hepáticos para análise histológica (um a quatro por caso) mediam cerca de $2 \times 1,5 \mathrm{~cm}$ e foram corados por hematoxilina e eosina. O diagnóstico de cirrose hepática baseou-se nos achados de fibrose sistematizada, com formação de septos delimitando nódulos de tamanhos variados, hiperplasia regenerativa de hepatócitos e inflamação crônica inespecífica (septal, periportal e lobular) ${ }^{(15)}$.

Para a análise do pâncreas, os fragmentos mediam aproximadamente $2 \times 1,5 \mathrm{~cm}$, variaram de um a três por caso e também foram corados por hematoxilina e eosina. Estabeleceu-se o diagnóstico histopatológico de pancreatite crônica em função da identificação de focos de exsudato de linfócitos, histiócitos e, eventualmente, plasmócitos (chamado exsudato mononuclear [EM]) em torno de ductos e no tecido conjuntivo inter e intralobular, acompanhados de traves de fibrose. Quando havia EM discreto, em região interlobular, periductal ou intralobular acompanhado de finas traves de fibrose, a pancreatite crônica foi classificada como leve. Pancreatite crônica moderada foi definida pelo aumento da intensidade do exsudato e da fibrose, ocasionalmente associados à pequena dilatação de ductos. A pancreatite crônica grave caracterizou-se por EM, densa fibrose, presença de rolhas protéicas, dilatação irregular dos ductos, atrofia glandular e, às vezes, calcificação intraductal|(34).

A presença de apenas EM nos cortes de pâncreas, sem a concomitância de fibrose, apesar de ser sugestiva de inflamação crônica, não foi considerada suficiente para o diagnóstico de pancreatite crônica, de acordo com os critérios aceitos internacionalmente para a definição histológica dessa doença ${ }^{(34)}$.

Entre os 25 pacientes portadores de CHA incluídos no trabalho, 13 (52\%) não tinham nos prontuários resultados de exames sorológicos para o vírus da hepatite $B$ (HBV). Dos 
outros 12 (48\%), 11 (44\%) apresentavam o exame HBsAg (método Elisa) negativo, e em um paciente (4\%), o resultado desse exame era positivo. Dezenove pacientes (76\%) não tinham sorologia para o vírus da hepatite $\mathrm{C}(\mathrm{HCV}) \mathrm{e}$ os outros seis possuíam o exame anti-HCV (método Elisa) negativo. Como nenhum paciente possuía soro estocado, optou-se por fazer a pesquisa do HBV no tecido hepático, através de imuno-histoquímica (IHQ), porém não foi possível localizar os blocos de fígado de sete dos 13 pacientes sem a sorologia para o HBV, os quais eram, na maioria, casos de necropsias mais antigas. Entre os seis pacientes que não tinham a sorologia para o HBV e tiveram o fígado examinado pelo teste imuno-histoquímico, cinco tiveram os resultados negativos e um, positivo. A reação de IHQ foi feita para o antígeno de superfície (HBsAg) e para o antígeno do core (HBcAg) (Sigma-Aldrich Co., St. Louis, USA). O sistema de amplificação do sinal foi realizado através do complexo Envision Plus Dako Envision labelled polymer peroxidase (Dako, Carpintaria, CA, USA). A identificação IHQ do antígeno core do HCV não foi testada devido à baixa sensibilidade inerente ao método, ou seja, capacidade de detectar a infecção em apenas um terço dos $\operatorname{casos}^{(1)}$.

Este projeto foi aprovado pelo Comitê de Ética em Pesquisa da UFU.

\section{Resultados}

Em 20 dos 25 (80\%) pacientes portadores de CHA constatou-se PC, considerada, histologicamente, leve em 13 (52\%), moderada em dois (8\%) e grave em cinco (20\%) pacientes. Os outros cinco (20\%), nos quais foram evidenciados apenas discretos focos de EM em torno dos ductos e no tecido conjuntivo inter ou intralobular, não foram considerados portadores de PC (Tabela 1). Entre os pacientes com $\mathrm{PC}$, o EM e a fibrose localizaram-se na região intralobular em $10(50 \%)$ e nas regiões intra e interlobular nos outros 10 (50\%) pacientes (Tabela 2). Alguns aspectos histológicos das pancreatites crônicas leve, moderada e grave são mostrados na Figura 1.

\section{Discussão}

Ao exame histológico, 20/25 (80\%) pacientes portadores de CHA apresentaram algum grau de PC no presente estudo, o que o difere de outros trabalhos que utilizando metodologia semelhante mostraram freqüências menores da doença pancreática. No Japão(22) foram encontrados

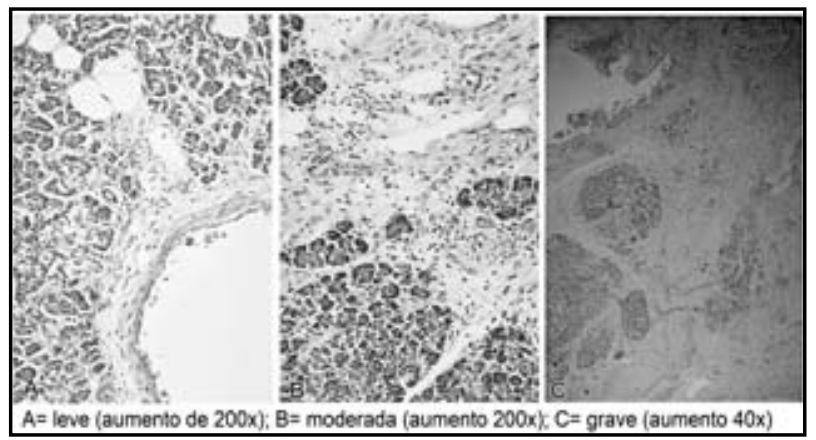

Figura 1 - Pancreatite crônica. A: leve; B: moderada; C: grave. Coloração: hematoxilina e eosina; aumentos originais de 200x, 200x e 40x, respectivamente

\section{Tabela 1}

Frequiência de pancreatite crônica, de acordo com a sua gravidade histológica, entre os

\begin{tabular}{lcccc} 
Tabela 1 & \multicolumn{4}{c}{ pacientes portadores de cirrose hepática alcoólica $(\boldsymbol{n}=\mathbf{2 5})$} \\
\hline \multicolumn{5}{c}{ Pancreatite crônica $(\%)$} \\
Ausente & Leve & Moderada & Grave & Total \\
$5(20)$ & $13(52)$ & $2(8)$ & $5(20)$ & $25(100)$ \\
\hline
\end{tabular}

\section{Tabela 2 hepática alcoólica $(n=20)$}

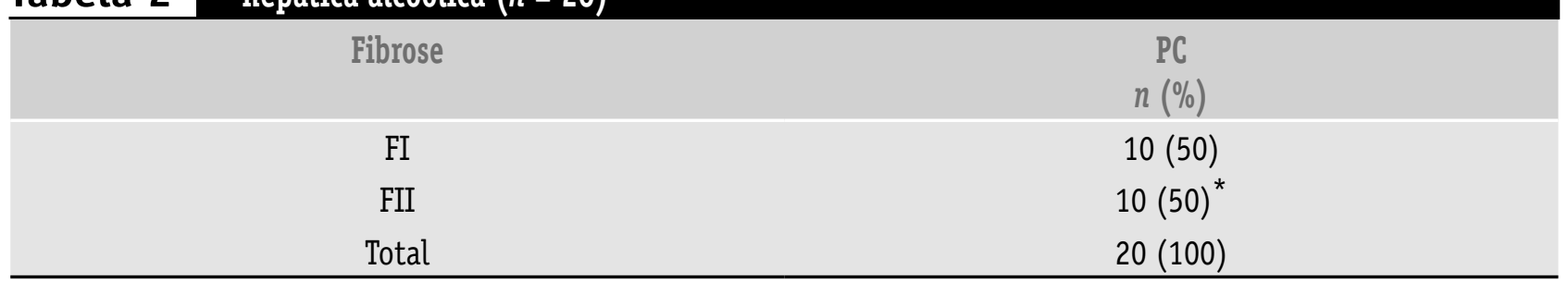

Fl: fibrose interlobular; Fll: fibrose inter e intralobular; ${ }^{*}$ cinco predominantemente inter e cinco predominantemente intralobular. 
apenas $8,9 \%$ da associação de PC e CHA, resultado que se pode dever ao fato, como os próprios autores relatam, de ser baixa a prevalência de pancreatite crônica de etiologia alcoólica naquele país, provavelmente em conseqüência de fatores genéticos e/ou nutricionais da população. Não é possível uma clara explicação para a divergência entre os resultados do presente estudo e aqueles observados em trabalhos realizados em outros países, nos quais se observaram freqüências de $20 \%{ }^{(34)}, 24 \%{ }^{(24)}$ e $46 \%{ }^{(39)}$ de $P C$ em pacientes com CHA, ainda que nos dois primeiros fossem utilizados critérios para o diagnóstico histológico de PC semelhantes aos deste estudo. No entanto, é lícito supor que possam contribuir para a alta freqüência de PC encontrada no presente estudo: fatores genéticos, nutricionais e até mesmo o tipo de bebida mais consumida em nosso meio, a cachaça, que possui elevada concentração de etanol.

Foi proposto que nos trabalhos em que se observam altos índices de coexistência de PC e CHA geralmente são incluídos pacientes mais velhos e, portanto, a fibrose encontrada, um dos parâmetros mais importantes para o diagnóstico de PC, poderia ser decorrente do próprio envelhecimento pancreático(2). Os pacientes com CHA deste estudo tinham idade média de 47,2 $\pm 13,8$ anos, variando de 24 a 83 anos. Observou-se que entre eles a distribuição e a freqüência de fibrose pancreática intra e interlobular foi semelhante, não havendo diferença entre os pacientes mais velhos e os mais jovens.

Em trabalhos nos quais se avaliou o pâncreas em pacientes portadores de CHA por outros métodos, foram encontrados resultados bastante variáveis em relação à concomitância das duas doenças. Utilizando-se a colangiopancreatografia endoscópica retrógrada (CPER) descreveuse essa concomitância em $7,7 \%^{(10)}, 18,8 \%^{(2)}, 19,7 \%^{(21)}$, $38,7 \% \%^{(23)}, 41,8 \%{ }^{(26)}$ e $63,6 \%{ }^{(32)}$ dos pacientes. Embora a CPER seja considerada um exame-padrão para o diagnóstico de pancreatite crônica, mostra apenas o sistema ductal do pâncreas, o qual pode ser normal ou de difícil interpretação em até $20 \%$ dos pacientes com a doença ${ }^{(7)}$.

No presente estudo, a PC foi caracterizada como leve em 13 (52\%) pacientes avaliados. Nessa forma pode não haver manifestações clínicas, e seu diagnóstico por exames de imagem é bastante difícil. A ultra-sonografia abdominal pode perder a sensibilidade tanto pela interposição de alças quanto pelo aumento da espessura do panículo adiposo do paciente, o que pode proporcionar até $25 \%$ de resultados falso-negativos ${ }^{(4)}$, enquanto a tomografia computadorizada de abdome, de modo geral, tem sensibilidade em torno de $75 \%$ para o diagnóstico de $\mathrm{PC}^{(6)}$. Isso poderia explicar a discrepância entre os resultados deste estudo e o baixo índice de diagnóstico de PC em pacientes com CHA nos trabalhos que avaliaram o pâncreas através de exames de imagem, principalmente em estágios iniciais da doença. Assim, os resultados dos exames de imagem nem sempre se relacionam com o verdadeiro estado da glândula, o qual poderia ser mais bem demonstrado por outros métodos, tais como a histopatologia ou testes funcionais, uma vez que a CPER e a tomografia podem ser normais em pacientes com diagnóstico histológico de $P C^{(36)}$. A avaliação do pâncreas de pacientes com CHA através de estudos funcionais $(S-C)$ mostrou freqüências de insuficiência pancreática exócrina de 17,3\%(40) e 40\%(2). Quando se utilizaram dois métodos de avaliação (um de imagem [CPER] e outro de pesquisa de gordura fecal) observou-se que, de 35 pacientes portadores de CHA, 11 $(31,4 \%)$ apresentavam alterações à CPER, e esteatorréia estava presente somente em dois casos (5,7\%), que apresentavam graves alterações à $\mathrm{CPER}^{(8)}$, demonstrando que a pesquisa de gordura fecal foi eficaz para o diagnóstico de PC somente na forma grave.

Não se dispôs de informes sorológicos e imuno-histoquímicos de sete (28\%) pacientes com $\mathrm{CH}$ em relação ao HBV e de 19 (76\%) ao HCV. Assim, em princípio, não se poderia rejeitar a hipótese de infecção viral crônica nesses indivíduos, concomitante ao pesado abuso crônico de álcool. Todos os pacientes sem marcadores de infecção pelo HBV tinham esteatose ao exame histopatológico do fígado, inclusive os sete que não foram examinados por $\mathrm{IHQ}$, e a esteatose hepática é muito freqüente nos pacientes com CH de etiologia alcoólica ${ }^{(3,15,38)}$. Além disso, 23 (92\%) pacientes tiveram cirrose hepática micronodular, que é o padrão morfológico mais comum de $\mathrm{CHA}^{(3,38)}$, e os dois pacientes (8\%) que apresentavam cirrose macronodular tinham sorologia negativa para o HBV, sendo que um deles também tinha esse exame negativo para o HCV. Um dos pacientes com $\mathrm{CH}$ tinha infecção ativa pelo HBV (HBsAg positivo) e outro, sem exame sorológico, teve a detecção imuno-histoquímica do vírus; o consumo médio diário de etanol desses dois pacientes, descrito em seus prontuários, era de $340 \mathrm{~g}$ por 10 anos e 18 anos, respectivamente. De qualquer modo, todos os pacientes tinham o diagnóstico de $\mathrm{CH}$ de etiologia alcoólica em vida, e com esse diagnóstico eram acompanhados ambulatorialmente, podendo-se supor que eles tivessem a investigação sorológica apenas não-anotada em seus prontuários, uma vez que a solicitação desses exames faz parte da rotina de avaliação de pacientes portadores de hepatopatias crônicas no HC/UFU. Também 
chamou a atenção que cinco (20\%) pacientes da casuística tinham PC grave, e esse diagnóstico não foi feito em vida. Isso deve ser salientado, uma vez que a PC grave pode contribuir para a desnutrição que freqüentemente é observada em pacientes portadores de cirrose hepática ${ }^{(35)}$.
Concluiu-se que, em nosso meio, a pancreatite crônica é um achado histopatológico muito freqüente em pacientes portadores de cirrose hepática alcoólica, tendo intensidade leve na maioria dos casos, mas podendo ser grave em 1/4 dos pacientes com essa doença.

\section{Referências}

I. ALVES, V.A.F. Hepatite C crônica: estudo de marcadores histológicos e imuno-histoquímicos do vírus e da resposta imune dos pacientes. Rev Soc Bras Med Trop, v. 31, p. 107-12, 1998.

2. ANGELINI, G. et al. Association of chronic alcoholic liver and pancreatic disease: a prospective study. Am J Gastroenterol, v. 80, p. 998-1003, 1985.

3. ARTEEL, G. et al. Advances in alcoholic liver disease. Best Pract Res Clin Gastroenterol, v. I7, p. 625-47, 2003.

4. BOLONDI, L. et al. Sonography of chronic pancreatitis. Radiol Clin North Am, v. 27, p. 8I 5-33, 1989

5. BOURLIERE, M. et al. Is tobacco a risk factor for chronic pancreatitis and alcoholic cirrhosis? Gut, v. 32, p. 1392-5, 1991.

6. BUSCAIL, L. et al. Endoscopic ultrasonography in chronic pancreatitis: a comparative prospective study with conventional ultrasonography, computed tomography, and ERCP. Pancreas, v. 10, p. 25।-7, 1995.

7. CALETTI, G. et al. Sensitivity of endoscopic retrograde pancreatography in chronic pancreatitis. Br J Surg, v. 69, p. 507-9, 1982.

8. CARADONNA, P. et al. Chronic pancreatitis prevalence in liver cirrhosis. Morphological and functional study. Ital J Gastroenterol, v. 28, p. 91-4, 1996.

9. CORRAO, G. et al. Alcohol consumption and micronutrient intake as risk factors for liver cirrhosis: a case-control study. Ann Epidemiol, v. 8, p. I54-9, 1998.

10. DEL OLMO MARTINEZ, M.L. et al. Función y morfología pancreática en el alcoholismo crónico con y sin cirrosis. Rev Esp Enf Dig, v. 82, p. 225-9, 1992.

I I. DENG, X. et al. Chronic alcohol-induced alterations in the pancreatic secretory control mechanisms. Dig Dis Sci, v. 49, p. 805-19, 2004.

12. DIEHL,A.M. Liver disease in alcohol abusers: clinical perspective. Alcohol, v. 27, p. 7-II, 2002.

13. DURBEC, J.P.; SARLES, H. Multicenter survey of the etiology of pancreatic diseases. Relationship between the relative risk of developing chronic pancreatitis and alcohol, protein and lipid consumption. Digestion, v. 18, p. 337-50, 1978.

14. FERNÁNDEZ-CHECA, J.C.; BELLENTANI, S.; TIRIBELLI, C. Alcohol-induced liver disease: from molecular damage to treatment. Rev Méd Chile, v. I 30, p. 681-90, 2002.

15. FLEMING, K.A.; McGEE, J.O'D. Alcohol-induced liver disease.J Clin Pathol, v. 37, p. 721-33, 1984.

16. FRANK, D.; RAICHT, R.F. Alcohol-induced liver disease. Alcohol Clin Exp Res, v. 9, p. 66-82, 1985.
17. GRANT, B.F; DUFOUR, M.C.; HARFORD,T.C. Epidemiology of alcoholic liver disease. Sem Liver Dis, v. 8, p. 12-25, 1988.

18. GRENDELL, J.H. Genetic factors in pancreatitis. Curr Gastroenterol Rep, v. 5, p. 105-9, 2003.

19. GULLO, L.Alcohol and chronic pancreatitis: leading or secondary etiopathogenetic role? J Pancreas (on line), v. 6, p. 68-72, 2005.

20. HANCK, C.; SCHNEIDER, A.; WHITCOMB, D.C. Genetic polymorphisms in alcoholic pancreatitis. Best Pract Res Clin Gastroenterol, v. 17, p. 613-23, 2003.

21. HASTIER, P. et al. A prospective study of pancreatic disease in patients with alcoholic cirrhosis: comparative diagnostic value of ERCP and EUS and long-term significance of isolated parenchymal abnormalities. Gastrointest Endosc, v. 49, p. 705-9, 1999.

22. ICHIHARA, S.; SATO, M.; KOZUKA, S. Prevalence of pancreatitis in liver diseases of various etiologies: an analysis of 107,754 adult autopsies in Japan. Digestion, v. 5I, p. 86-94, 1992.

23. $\mathrm{KOCHHAR,} \mathrm{R.} \mathrm{et} \mathrm{al.} \mathrm{Concurrent} \mathrm{pancreatic} \mathrm{ductal} \mathrm{changes}$ in alcoholic liver disease. J Gastroenterol Hepatol, v. I8, p. I067-70, 2003.

24. MARIN, G.A; CLARK, M.L.; SENIOR, J.R. Studies of malabsorption occurring in patients with Laennec's cirrhosis. Gastroenterology, v. 56, p. 727-36, 1969.

25. MINCIS, M. Doença hepática alcoólica. In: Gastroenterologia e hepatologia: diagnóstico e tratamento. 3. ed. São Paulo: Lemos Editorial, 1997. Cap. 64; p. 695-7I6.

26. NAKAMURA, Y. et al. Severe chronic pancreatitis and severe liver cirrhosis have different frequencies and are independent risk factors in male Japanese alcoholics. J. Gastroenterol, v. 39 , p. 879-87, 2004.

27. NEVES, M.M; BORGES, D.R.; VILELA, M.P. Concentração de etanol em bebidas alcoólicas mais consumidas no Brasil. GED, v. 8, p. 17-20, 1989.

28. NOEL-JORAND, M.C; BRAS, J. A comparison of nutritional profiles of patients with alcohol-related pancreatitis and cirrhosis. Alcohol Alcohol, v. 29, p. 65-74, 1994.

29. NORTON, R. et al. Alcohol consumption and the risk of alcoholrelated cirrhosis in women. BrMed J, v. 295, p. 80-2, 1987.

30. PARODI, H.C. et al. Pancreatitis crónica calcificada. Nuestra experiencia. Acta Gastroenterol Latinoam, v. 14, p. I-12, 1984.

31. PITCHUMONI, C.S. Chronic pancreatitis: a historical and clinical sketch of the pancreas and pancreatitis. Gastroenterologist, v. 6, p. 24-33, 1998. 
32. RAMIREZ DEGOLLADO, J. et al. Alteraciones pancreáticas en los pacientes con cirrosis hepática por alcoholismo. Rev Gastroenterol Mex, v. 46, p. 159-62, 1981.

33. REED, T. et al. Genetic predisposition to organ-specific endpoints of alcoholism. Alcohol Clin Exp Res, v. 20, p. 1528-33, 1996.

34. RENNER, I.G. et al. Pancreatitis associated with alcoholic liver disease: a review of 1,022 autopsy cases. Dig Dis Sci, v. 29, p. 593-9, 1984.

35. ROONGPISUTHIPONG, C. et al. Nutritional assessment in various stages of liver cirrhosis. Nutrition, v. 17, p. 76I-5, 2001.

36. SARNER, M.; COTTON, P.B. Classification of pancreatitis. Gut, v. 25, p. 756-9, 1984.

37. SAUNDERS, J.B; DAVIS, M.;WILLIAMS, R. Do women develop alcoholic liver disease more readily than men? Br Med J, v. 282, p. $1140-3,1981$.

38. SHERLOCK, S.; DOOLEY, J. Alcohol and the liver. In: Diseases of the liver and biliary system. I I th ed. Oxford: Blackwell Science Ltd., 2002. p. 38I-98.

39. SOBEL, H.J.; WAYE, J.D. Pancreatic changes in various types of cirrhosis in alcoholics. Gastroenterology, v. 45, p. 34l-4, 1963.

40. SOFIA, C. et al. Coexistência de doença hepática e pancreática no alcoólico crônico. Situação rara ou freqüente? Acta Med Port, v. 5, p. 235-8, 1992.

4I.TAKADA, A.;TAKASE, S.;TSUTSUMI, M. Characteristic features of alcoholic liver disease in Japan: a review. Gastroenterol Jpn, v. 28, p. I37-48, 1993. 